\title{
A DUALITY FOR CERTAIN DIFFERENCE EQUATIONS
}

\author{
E. T. BELL
}

1. Umbral factorials and Appell polynomials. The difference equations considered include as special cases those satisfied by the generalized Bernoulli and Euler polynomials, ${ }^{*}$ also many of interest in the theory of numbers; the duality was primarily devised for the investigation of divisibility properties of certain sequences of rational numbers obtained from generalizations of the polynomials mentioned. The connection with arithmetic is made through Fermat's theorem and Lagrange's identical congruence, and is developed elsewhere. $\dagger$ In constructing the duality, an inconsistency in the usual statement of symbolic equations that has been overlooked by writers on the symbolic methods is uncovered ( $\$ 1$, end). This inconsistency is trivial for the customary uses of the symbolic method, but until it is rectified it is impossible to proceed to the new applications of the method made here.

The duality is most simply displayed in Blissard's symbolism (suitably amplified), in which $\alpha$ is the umbra of the sequence $\alpha_{n}$, $(n=0,1, \cdots)$, or of the vector $\left(\alpha_{0}, \alpha_{1}, \cdots\right)$, and the symbolic power $\alpha^{n}$ denotes $\alpha_{n}$. Small Greek letters without suffixes, $\alpha, \alpha^{(n)}$, $\beta, \beta^{(n)}, \cdots$, and $S^{(n)}$, will be used exclusively for umbrae, small Latin letters, with or without suffixes, $a, a_{i}, \cdots, x, x_{i}, t, \cdots$ for ordinaries (real or complex numbers). Small Greek letters with suffixes, as $\alpha_{n}, \alpha_{r}^{(n)}, \cdots$, denote ordinaries. By definition, $x \alpha$ is the umbra of $x^{n} \alpha_{n},(n=0,1, \cdots)$. Umbral equality, $\alpha=\beta$, signifies $\alpha^{n}=\beta^{n}$, that is, $\alpha_{n}=\beta_{n},(n=0,1, \cdots)$. If $\alpha \neq \beta, \alpha, \beta$ are said to be distinct.

Provided the series converges for some $|t|>0$,

$$
e^{x \alpha t} \equiv \sum_{n=0}^{\infty} x^{n} \alpha^{n} \frac{t^{n}}{n !} \equiv \sum x^{n} \alpha_{n} \frac{t^{n}}{n !} .
$$

Hence, if $\alpha, \beta, \cdots, \gamma$ are all distinct, and $x y \cdots z \neq 0$,

$$
e^{x \alpha t} e^{y \beta t} \cdots e^{z \gamma t}=e^{(x \alpha+y \beta+\cdots+z \gamma) t},
$$

convergence of the umbral exponentials on the left being assumed, where $(x \alpha+y \beta+\cdots+z \gamma)^{s},(s \geqq 0)$, is to be replaced after expansion

* N. E. Nörlund, Differenzenrechnung, chap. 6.

$\dagger$ In a paper on generalized Stirling transforms of sequences to appear in the American Journal of Mathematics, 1939. 
of the right by its equivalent as given by the umbral multinomial theorem,

$$
\begin{aligned}
& (x \alpha+y \beta+\cdots+z \gamma)^{0}=\alpha_{0} \beta_{0} \cdots \gamma_{0}, \\
& (x \alpha+y \beta+\cdots+z \gamma)^{s}=\sum M_{a, b, \cdots, c} x^{a} y^{b} \cdots z^{c} \alpha_{a} \beta_{b} \cdots \gamma_{c}, s>0,
\end{aligned}
$$

in which the $M$ 's are multinomial coefficients from the ordinary multinomial theorem

$$
(x+y+\cdots+z)^{s}=\sum M_{a, b, \cdots, c} x^{a} y^{b} \cdots z^{c} .
$$

The exponents 0,1 of umbrae are treated precisely as any other nonnegative exponents, in distinction to the like in a field, where $x^{0}=1$, $x^{1}=x$. Thus $\alpha^{0}=\alpha_{0}, \alpha^{1}=\alpha_{1}$.

The umbrae $\eta, \omega, \delta^{(s)}, S^{(s)}$ are special:

(1) $\eta_{0}=1, \eta_{n}=0,(n>0) ; \omega_{n}=0,(n \geqq 0)$;

(2) $\delta^{(s)}$ is the umbra of $\left(\delta_{0}^{(s)}, \delta_{1}^{(s)}, \cdots\right)$, where $\delta_{r}^{(s)}$ is a Kronecker delta, $\delta_{s}^{(s)}=1, \delta_{r}^{(s)}=0,(r \neq s)$.

The (ordinary) factorial $(t)_{n}$ is defined by

$$
(t)_{0}=1, \quad(t)_{n} \equiv \prod_{s=0}^{n-1}(t-s)=\sum_{s=1}^{n} S_{n}^{(s)} t^{s}, \quad n>0,
$$

where the $S_{n}^{(s)}$ are the Stirling numbers of the first kind, $S_{0}^{(0)}=1$, $S_{n}^{(s)}=0,(s>n)$. If $|t|<1$, there is the known expansion

$$
[\log (1+t)]^{s}=s ! \exp \left[S^{(s)} t\right]
$$

in which the $n$th power $(n \geqq 0)$ of $S^{(s)}$ is replaced after expansion by $S_{n}^{(s)}$

For any umbra $\xi$ the (umbral) factorial $(\xi)_{n}$ is defined by

$$
(\xi)_{0} \equiv \xi_{0}, \quad(\xi)_{n} \equiv \prod_{s=0}^{n-1}(\xi-s)=\sum_{s=1}^{n} S_{n}^{(s)} \xi_{s}, \quad n>0
$$

The umbra of $(\xi)_{n},(n=0,1, \cdots)$, is written $(\xi)$. If for some $|t|>0$ the series converge,

$$
(1+t)^{\xi}=e^{(\xi) t}
$$

where the left is expanded formally by the binomial theorem (as if $\xi$ were ordinary), the right by the exponential theorem, and $\xi^{n}$, $(\xi)^{n}$ are replaced after expansion by $\xi_{n},(\xi)_{n},(n \geqq 0)$.

The (ordinary) Appell polynomial $A_{n}(t, \alpha)$ in $t$ with the base $\alpha$ is $A_{0}(t, \alpha) \equiv \alpha_{0}$

$$
A_{n}(t, \alpha) \equiv(t+\alpha)^{n}=\sum_{s=0}^{n}(n, s) \alpha_{s} t^{n-s}, \quad n>0,
$$


where $(n, s)$ is the binomial coefficient $n ! / s !(n-s)$ !. The (umbral) Appell polynomial $A_{n}(\xi, \alpha)$ in $\xi$ with the base $\alpha$ is $A_{0}(\xi, \alpha) \equiv \alpha_{0} \xi_{0}$,

$$
A_{n}(\xi, \alpha) \equiv(\xi+\alpha)^{n}=\sum_{s=0}^{n}(n, s) \alpha_{s} \xi_{n-s}, \quad n>0 ;
$$

whence $A(\xi, \alpha)=A(\alpha, \xi)$. If $\tau$ is the umbra of $\left(1, t, t^{2}, \cdots\right)$, then $A(\tau, \alpha)=A(t, \alpha)$. Thus the umbral Appell polynomials generalize the ordinary ones.

Let $\xi$ be distinct from $\alpha, \beta$. Umbral derivation, $D_{\xi}$, with respect to $\xi$ is defined by

$$
\begin{array}{ll}
D_{\xi} \alpha_{m} \xi_{n}=\alpha_{m} D_{\xi} \xi_{n}=n \alpha_{m} \xi_{n-1}, & n>0, \\
D_{\xi} \alpha_{m} \xi_{0}=0, \quad D_{\xi}\left(\alpha_{m} \xi_{n}+\beta_{r} \xi_{s}\right)=D_{\xi} \alpha_{m} \xi_{n}+D_{\xi} \beta_{r} \xi_{s} . &
\end{array}
$$

Hence, generalizing the characteristic property of Appell polynomials, we have $D_{\xi} A_{0}(\xi, \alpha)=0$,

$$
D_{\xi} A_{n}(\xi, \alpha)=n A_{n-1}(\xi, \alpha), \quad n>0 .
$$

We have also, by (1),

$$
A(\xi, \eta)=\xi, \quad A(\xi, \omega)=\omega .
$$

In the definition of $(\xi)$ as the umbra of the sequence $(\xi)_{n}$, $(n=0,1, \cdots)$, of umbral factorials constructed from $\xi, \xi$ is arbitrary. Hence the umbra of the sequence $(A(\xi, \alpha))_{n},(n=0,1, \cdots)$, of factorials constructed from $A(\xi, \alpha)$ is written $(A(\xi, \alpha))$. In the definition (4), $\xi$ is any umbra. Hence, replacing $\xi$ in (4) by $A(\xi, \alpha)$, we have $(A(\xi, \alpha))_{0}=\xi_{0} \alpha_{0}$,

$$
(A(\xi, \alpha))_{n}=\sum_{s=1}^{n} S_{n}^{(s)} A_{s}(\xi, \alpha), \quad n>0 .
$$

It may be shown from the properties of the Stirling numbers that

$$
(A(\xi, \alpha))_{n}=[(\xi)+(\alpha)]^{n}, \quad n=0,1, \cdots ;
$$

that is, by the definition of umbral equality, we have the second of the next two equations,

$$
A(\xi, \alpha)=\xi+\alpha, \quad(A(\xi, \alpha))=(\xi)+(\alpha),
$$

the first of which is equivalent to (6). Thus the first of (10) implies the second. It can be shown in the same way that the second implies the first.

An implied restriction on ordinary constants occurring in equations containing umbrae appears to have been overlooked by writers on 
the umbral calculus, as its necessity for consistency can be ignored until the umbral equations are dualized as in the next section. The point is sufficiently evident from an example. Let $E$ be the umbra of the Euler numbers. Then one of the usual umbral equations for the $E_{n}$ is customarily written

$$
(E+2)^{n}+E^{n}=2, \quad n=0,1, \cdots .
$$

The terms on the left are umbrae, the constant 2 on the right is an ordinary, and it is meaningless to equate an umbra to an ordinary, although the (meaningless) equation gives correct results in the classic applications of the symbolic method. The equation is derived from

$$
\operatorname{sech} t=2 e^{t} /\left(e^{2 t}+1\right) \equiv e^{E t},
$$

which may be taken as a definition of $E$. Proceeding as usual we get

$$
e^{(E+2) t}+e^{E t}=2 e^{t}
$$

and hence, on equating coefficients of $t$,

$$
(E+2)^{n}+E^{n}=2 \cdot 1^{n},
$$

the correct form, in which 2 appears as $2 \cdot 1^{n}$. The 1 in $1^{n}$ is the umbra of $\left(1^{0}, 1^{1}, 1^{2}, \cdots\right)$, namely of $(1,1,1, \cdots)$; and it is clear why the incorrect equation gives correct results. Generally, if an equation involving umbrae contains also an ordinary term $c$, this term must be interpreted as the umbra of $\left(c, c 1, c 1^{2}, \cdots\right)$. Similarly, in $\xi+c, c$ is the umbra of $\left(1, c, c^{2}, \cdots\right)$.

2. Duality. Two relations, each of which implies the other, will be called duals. The dual difference equations considered are obtained as follows. Let $\alpha^{(r)}, \beta^{(s)}, \cdots$ be all the umbrae other than the special umbrae $\delta^{(u)}, S^{(v)}$, or umbrae of the type noted at the end of the preceding section, occurring in the first equation of a pair of duals. Let the first equation be between $n$th powers of umbrae, valid for $n=0,1, \cdots$. Then passage from the first equation to the second is made by the substitution

$$
\alpha^{(r)} \rightarrow\left(\alpha^{(r)}\right), \beta^{(s)} \rightarrow\left(\beta^{(s)}\right), \cdots,
$$

and, if one or more of $\delta^{(u)}, S^{(v)}, c a^{n}$ occurs, by the corresponding substitutions from among

$$
\delta^{(u)} \rightarrow S^{(u)}, \quad S^{(v)} \rightarrow \delta^{(v)}, \quad c a^{n} \rightarrow c(a)_{n} .
$$

Passage from the second equation to the first is made by the inverse substitutions 


$$
\begin{aligned}
\left(\alpha^{(r)}\right) & \rightarrow \alpha^{(r)}, & \left(\beta^{(s)}\right) & \rightarrow \beta^{(s)}, \cdots, \\
S^{(u)} & \rightarrow \delta^{(u)}, & \delta^{(v)} & =S^{(v)}, \quad c(a)_{n} \rightarrow c a^{n} .
\end{aligned}
$$

For example, $E$ being as in $\$ 1$, end, we have the duals

$$
\begin{aligned}
(E+2)^{n}+E^{n} & =2 \cdot 1^{n}, \\
{[(E)+(2)]^{n}+(E)^{n} } & =2(1)_{n}, \quad n \geqq 0 .
\end{aligned}
$$

Now $(c)_{0}=1,(c)_{n}=c(c-1) \cdots(c-n+1),(n>0)$. Hence $(2)_{0}=1$, $(2)_{1}=(2)_{2}=2 ;(2)_{n}=0,(n>2) ;(1)_{0}=1,(1)_{n}=0,(n>1) ;$ and since

$$
[(E)+(2)]^{n}=\sum_{s=0}^{n}(n, s)(E)_{n-s}(2)_{s}
$$

the above duals in expanded form are

$$
E_{0}=1, \quad E_{n}+\sum_{s=1}^{n} 2^{s-1}(n, s) E_{n-s}=1, \quad n>0 ;
$$

$(E)_{0}=1,2(E)_{n+2}+2(n+2)(E)_{n+1}+(n+1)(n+2)(E)_{n}=0, n \geqq 0$.

The solution of the last is $(E)_{n}=n ! a_{n},(n \geqq 0)$, where

$$
a_{4 n}=(-1)^{n} 2^{-2 n}, a_{4 n+1}=0, a_{4 n+2}=(-1)^{n+1} 2^{-2 n-1}, a_{4 n+3}=(-1)^{n} 2^{-2 n-1} \text {. }
$$

With the definitions $0^{0}=(0)_{0}=1$, we have also the duals

$$
\begin{aligned}
(E+1)^{n}+(E-1)^{n} & =2 \cdot 0^{n}, \\
{[(E)+(1)]^{n}+[(E)+(-1)]^{n} } & =2(0)_{n}, \quad n \geqq 0 .
\end{aligned}
$$

3. Duals. We shall give one pair, $(15),\left(15^{\prime}\right)$, of duals of a very general character. Proofs are deferred for the moment.

The $\eta, \omega, \delta^{(n)}, S^{(n)}$ have the special meanings assigned in $\$ 1$; $h, i, j, k$ are nonnegative integers ranging, respectively, from 0 to $l$, 0 to $m, 0$ to $n, 0$ to $r ; L_{h}, M_{i}, N_{j}, R_{k}$ are any given ordinary constants, $\lambda^{(h)}, \mu^{(i)}, \nu^{(j)}, \rho^{(k)}$ any given umbrae such that

$$
N_{0} \nu_{0}^{(0)}+\sum_{k=0}^{r} R_{k} \rho_{0}^{(k)} \neq 0 .
$$

It follows that the $\theta_{n},(n=0,1, \cdots)$, given by

$$
\begin{aligned}
\sum_{j=0}^{n} j ! N_{j}\left[\theta+A\left(\nu^{(j)}, \delta^{(j)}\right)\right] u & +\sum_{k=0}^{r} R_{k}\left[\theta+\rho^{(k)}\right]^{u} \\
& =\sum_{h=0}^{l} h ! L_{h} A_{u}\left(\lambda^{(h)}, \delta^{(h)}\right)+\sum_{i=0}^{m} M_{i} \mu_{u}^{(i)},
\end{aligned}
$$


for $u=0,1, \cdots$, are determinate when, by convention, the binomial coefficient $(0,0)=1,(0, s)=0,(s>0)$.

The dual $\left(12^{\prime}\right)$ of $(12)$ is the equation satisfied by $(\theta)$. It is written down from (12) by the substitution

$$
\begin{aligned}
\theta, \nu^{(j)}, \rho^{(k)}, \lambda^{(h)}, \mu_{u}^{(i)} & \rightarrow(\theta),\left(\nu^{(j)}\right),\left(\rho^{(k)}\right),\left(\lambda^{(h)}\right),\left(\mu^{(i)}\right)_{u} ; \\
\delta^{(j)}, \delta^{(h)} & \rightarrow S^{(j)}, S^{(h)} .
\end{aligned}
$$

An alternative form $\left(12^{\prime \prime}\right)$ of $\left(12^{\prime}\right)$ is given from $\left(12^{\prime}\right)$, by the second equation of (10), by the substitution

$$
\begin{aligned}
\theta, \mu^{(i)}, \rho^{(k)} & \rightarrow(\theta),\left(\mu^{(i)}\right),\left(\rho^{(k)}\right) ; \\
A\left(\nu^{(j)}, \delta^{(j)}\right), A\left(\lambda^{(h)}, \delta^{(h)}\right) & \rightarrow\left(A\left(\nu^{(j)}, \delta^{(j)}\right)\right),\left(A\left(\lambda^{(h)}, \delta^{(h)}\right)\right) .
\end{aligned}
$$

We now generalize (12), $\left(12^{\prime}\right),\left(12^{\prime \prime}\right)$ by constructing the equations satisfied by the umbral Appell polynomials $A_{n}(\xi, \theta),(n=0,1, \cdots)$, in $\xi$ with the base $\theta$, and by the factorials $(A(\xi, \theta))_{n}$ constructed from the $A_{n}(\xi, \theta)$. The equation for $A(\xi, \theta)$ can be written down from (12); that for $(A(\xi, \theta))$ is then obtained immediately by dualizing. We find

$$
\begin{gathered}
\sum_{j=0}^{n} j ! N_{j}\left[A(\xi, \theta)+A\left(\nu^{(j)}, \delta^{(j)}\right)\right]^{u}+\sum_{k=0}^{r} R_{k}\left[A(\xi, \theta)+\rho^{(k)}\right]^{u} \\
=\sum_{h=0}^{l} l ! L_{h}\left[\xi+A\left(\lambda^{(h)}, \delta^{(h)}\right)\right]^{u}+\sum_{i=0}^{m} M_{i}\left[\xi+\mu^{(i)}\right]^{u}, \\
u=0,1, \cdots .
\end{gathered}
$$

This follows from (12) by the first equation of (10) on replacing $\theta$ in (12) by $\xi+\theta$, expanding the result in powers of $\xi$, and using (12) with $u=0,1, \cdots$ to identify coefficients of $\xi_{u}$ in (15). As already noted, $A_{n}(\xi, \theta)$ is the ordinary Appell polynomial $A_{n}(x, \theta)$ in $x$ with the base $\theta$ if $\xi$ is its special case, the umbra of $\left(1, x, x^{2}, \cdots\right)$. To obtain (12) from (15) we take $\xi \equiv \eta$ and refer to the first of (8). The dual (15') of (15) may be stated in each of several equivalent forms. One is obtained by the substitution

$A(\xi, \theta), A\left(\nu^{(j)}, \delta^{(j)}\right), A\left(\lambda^{(h)}, \delta^{(h)}\right) \rightarrow(A(\xi, \theta)),\left(A\left(\nu^{(j)}, \delta^{(j)}\right)\right),\left(A\left(\lambda^{(h)}, \delta^{(h)}\right)\right)$

in (15), with $\rho^{(k)} \rightarrow\left(\rho^{(k)}\right)$ on the left and $\xi, \mu^{(i)} \rightarrow(\xi),\left(\mu^{(i)}\right)$ on the right. We prove the foregoing results first under a convergence restriction which will be removed presently. Assume for a moment that all the expressions

$$
\exp \lambda^{(h)} t, \quad \exp \mu^{(i)} t, \quad \exp \nu^{(j)} t, \quad \exp \rho^{(k)} t
$$

when written out as ordinary MacLaurin series are absolutely con- 
vergent for some $|t|>0$. By (11), the like is true for $Q(t) \equiv F(t) / G(t)$, where

$$
\begin{aligned}
& F(t) \equiv \sum_{h=0}^{l} L_{h} t^{h} \exp \lambda^{(h)} t+\sum_{i=0}^{m} M_{i} \exp \mu^{(i)} t \\
& G(t) \equiv \sum_{j=0}^{n} N_{j} t^{i} \exp \nu^{(j)} t+\sum_{k=0}^{r} R_{k} \exp \rho^{(k)} t
\end{aligned}
$$

Writing $Q(t) \equiv \exp \theta t$, thus defining $\theta$, we have

$$
t^{h}=h ! \exp \delta^{(h)} t, \quad t^{j}=j ! \exp \delta^{(j)} t ;
$$

and equating coefficients of $\theta^{u}$ in the identity in $t, Q(t) G(t) \equiv F(t)$, we get (12). Again,

$$
e^{\xi t} Q(t)=e^{(\xi+\theta) t} \equiv\left[e^{\xi t} F(t)\right] / G(t)
$$

hence

$$
G(t) \exp A(\xi, \theta) t=F(t) \exp \xi t ;
$$

whence (15) follows. The duals are obtained by the substitution $t \rightarrow \log (1+t)$, obviously permissible under the convergence assumptions, in the foregoing identities, and (3), (5). By the inverse substitution $t \rightarrow e^{t}-1$, the equivalence of the duals follows. To remove the convergence restriction we refer to a former paper* where it was proved that in such formal uses of power series as are sufficient here convergence is irrelevant.

To save space, all applications, of which several have been made, will be omitted here, but will appear elsewhere.

California Institute of Technology

* Transactions of this Society, vol. 25 (1923), pp. 135-154, §2. 